\title{
Universidade, contexto ansiogênico? Avaliação de traço e estado de ansi edade em estudantes do ciclo básico
}

\author{
Evaluation of trait and state anxiety in first year students
}

\author{
Camomila Lira Ferreira ${ }^{1}$ \\ Katie M oraes de Almondes ${ }^{1}$ \\ Liliane PereiraBraga ${ }^{1}$ \\ Ádala Nayana de Sousa M ata ${ }^{1}$ \\ CarolineAraújo Lemos ${ }^{1}$ \\ Eulália M aria Chaves M aia ${ }^{1}$
}

Abstract The purpose of this study is to evaluate trait anxiety and state anxiety in first year students from theFederal U niversi ty of Rio Grande do N orte, verifying differences between three great areas of knowledge - Biomedical, Humanistic and Technological. The entrance to a University can be perceived as a threatening situation that seems to suffer influence from the different characteristics of each of these areas. 158 students, 71 females and 87 males aged 20.04 \pm 3,37 years, answered an I dentification Form and the State and Trait of Anxiety Inventory (STAI). The means of trait anxiety and state anxiety observed in these students were as expected for this population although the Biomedical area is perceived as the most anxiogenic for having a dense curriculum with intense academic demands, what seems to increase the scores of the students of this area, especially of the men.

Key words Trait anxiety, State anxiety, First year university students, $\mathrm{H}$ ealth psychology
${ }^{1}$ Grupo de Estudos:

Psicologia e Saúde (GEPS), Departamento de

Psicologia, Universidade

Federal do Rio Grande do Norte. Campus

Universitário, Lagoa Nova. 59078-970 Natal RN. artemisiadesouza@ yahoo.com.br
Resumo 0 presente artigo objetiva avaliar a ansiedade traço ea ansiedade estado de estudantes universitários do ciclo básico da Universidade Federal do Rio Grande do N orte, verificando diferenças entre três grandes áreas de conheci mento, bi omédica, humanística e tecnológica, tendo em vista que a entrada na universidade pode se configurar como uma situação ameaçadora que parece sofrer influência das diferentes características de cada uma dessas áreas. Participaram do estudo 158 estudantes, sendo 71 mulherese 87 homens, com idade média de20,04 $\pm 3,37$ anos, respondendo uma ficha de identificação eo Inventário de Ansi edade Traço-Estado (IDATE) . Resultados demonstram queas médias deansiedade traço e ansi edade estado desses estudantes encontram-sedentro do esperado para essa população, embora a área biomédica seja percebi da como a mais ansiogênica, uma vez queapresenta uma densa grade curricular com intensas demandas acadêmicas, 0 que pareceelevar os escores dos estudantes dessa área, em especial os dos homens.

Palavras-chave Ansiedade-traço, Ansiedadeestado, Estudantes universitários, Ciclo básico, Psicologia da saúde 
Introdução

A ansiedade é considerada um sinal de alerta, determinada pela presença de um conflito interno, cuja função éavisar sobreum perigo iminentepara que se tome medidas para lidar com a ameaça. É certo quetodas as pessoas já vivenciaram ansiedade, seja como resposta normal e adaptativa aos estímulos, ou como uma sensação desajustadora e patológica1,2.

$A$ ansiedade tem aumentado expressivamente na população humana no último século, sobretudo devido às profundas transformações ocorridas no âmbito econômico, social e cultural. Essas mudanças acabaram por exigir que a população se adaptasse a um novo ritmo de vida, tornando o século XX conhecido como a era da ansiedade ${ }^{3}$. Desde então, esta sensação tem sido investigada e relacionada diretamente à situação vivenciada pelos indivíduos em seu cotidiano eàs exigências decorrentes dela ${ }^{4-6}$.

Tais investigações apresentam-se de extrema importância e relevância nos meios científicos e assistenciais, na medida em quea ansiedade está vinculada a sintomas como taquicardia, tontura, dor de cabeça, dores musculares, formigamento, suor, além de insônia, tensão, irritabilidade e angústia. A presença ea intensidade desses sinais podem trazer consequências prejudiciais paraas condições devidaede saúde da população em geral, uma vez que níveis elevados de ansiedade podem provocar percepções negativas quanto às habilidades motoras eintelectuais do indivíduo. Isso, por sua vez, interferena atenção seletiva e na codificação de informações na me mória, bloqueando a compreensão e o raciocínio7.

Estudantes universitários são um exemplo de população em que a ansiedade vem sendo estudada e relacionada à situação vivenciada. Silver ${ }^{8} \mathrm{de}$ monstra que, ao ingressarem na faculdade, os estudantes são submetidos a uma grande carga de estresse, devido a longas horas de estudo e cobranças pessoais de professores e familiares. Além disso, as transformações maturacionais (fisiológicas, neurológicas e psicológicas), decorrentes da transição entre a fase da adolescente e a fase de adulto, levam os estudantes a vivenciarem um período de crise, por exigir a adaptação a um novo papel social, o de adulto jovem ${ }^{9,10}$.

Assim, percebe-se a influência de duas principais circunstâncias no estado emocional dos estudantes: a entrada na universidade (situação considerada ansiogênica) e a passagem de uma fase de desenvolvimento para outra (situação considerada crise normativa). Essas circunstâncias podem ser associadas à ansiedade-estado e à ansiedade-traço.
A ansiedade-estado está ligada a um momento ou situação particular, como na entrada a universidadepel os estudantes, causando um estado emocional transitório. Já a ansiedadetraço está relacionada a características individuais e disposicionais, estabelecendo diferenças entreos indivíduos quanto à forma de encarar eventos diversos, ou seja, cada indivíduo traz consigo uma disposição maior ou menor de encarar as situações como ansiogênicas, estando relacionada, diretamente, à personalidade de cada um.

Na situação decrise, pela transição deum período de desenvolvimento para outro, no momento de traçar estratégias que possibilitem a superação desse período de crise, alguns indivíduos podem experienciar maisansiedade do queoutros deacordo com sua ten dência para encarar situações como ansiogênicas ${ }^{2,11}$. N os casos de el evação de ansiedade a níveis patológicos, esses indivíduos podem contar com o auxílio dos profissionais da saúde mental (psicólogosepsiquiatras) nas redes deatenção básica à saúde, vinculadas ao Sistema Ú nico de Saúde, a fim de tratar tal elevação e de prevenir possíveis transtornos depressivos e outras doenças físicas associadas.

Alguns dados de literatura mostram análises da relação entre traço e estado de ansiedade e a situação vivenciada pelos estudantes universitários. Uma pesquisa com estudantes do curso de M edicina da Universidade Federal do Rio Grande do Norte (UFRN), por exemplo, avaliou o estado de ansiedade e o traço de ansiedade, em dois momentos do curso: quando assistiam aulas e cumpriam as demandas acadêmicas, e quando, além de assistirem aulas, enfrentavam plantões médicos. Resultados mostraram que o estado de ansiedade teve aumento estatisticamente significante no decorrer dos anos. Além disso, a ansiedade aumentou tanto para quem apresentava personalidade ansiosa (traço de ansiedade elevado) quanto para quem não apresentava essa característica ${ }^{12}$.

Em uma outra investigação, foi avaliada a ansiedade traço e a ansiedade-estado dos estudantes de Psicologia da UFRN, em três momentos do curso: o primeiro período, momento de ingresso no curso; 0 oitavo período, momento de escolha da área deatuação profissional; eo nono período, momento de realização dos estágios curriculares. Observouse que os estudantes do primeiro ano apresentaram escores acima da média esperada para estudantes universitários do ciclo básico, mostrandose muito ansiosos, tanto com relação à situação momentânea, como às características disposicionais da população estudada ${ }^{13}$.

É importante salientar que esses fatores ansio- 
gênicos podem interferir negativamente sobre alguns aspectos cognitivos, como o processo deaprendizagem, a redução de aten ção e da concentração, diminuindo, assim, a aquisição de habilidades ${ }^{14}$.

Assim, 0 objetivo da presente pesquisa foi avaliar a ansiedade-traço e ansiedade-estado dos estudantes do ciclo básico em vários cursos de graduação das três grandes áreas de conhecimento (humanística, tecnológica ebiomédica) da Universidade Federal do Rio Grande do Norte (UFRN), com diferentes características, avaliando se existiam diferenças entre os escores de ansiedade dessas áreas. I sto se faz importante em virtude da vivência da entrada na universidade pelos estudantes, que envolve circunstâncias geradoras de tensão, tais como: responsabilidades que serão assumidas exclusivamente por eles; demandas acadêmicas intensas; maiores exigências sobre seu desempenho acadêmico, proveniente não só deles, mas de seus colegas de sala, de seus professores e familiares.

\section{Métodos}

$$
\text { Sujeitos }
$$

Participaram do estudo 158 estudantes, com idade média de 20,04 $\pm 3,37$ - o que os classifica como adultos jovens - cursando o primeiro período de dezessete cursos das três áreas de conhecimento da UFRN : humanística, biomédica etecnológica. Destes estudantes, 53 eram da área humanística (35 mulheres e 18 homens), com média de idade de 19,72 $\pm 1,73$ anos, e pertencentes aos seguintes cursos: Administração, Ciências Econômicas, Turismo, Direito, Pedagogia e de Serviço Social. Dos alunos de biomédica, houve a participação de 47 sujeitos ( 25 mulheres e 22 homens), com média de idade de 21,04 $\pm 5,18$ anos. Estes estudantes pertenciam aos seguintes cursos: Ciências Biológicas, Enfermagem e Obstetrícia, Farmácia, $\mathrm{M}$ edicina e Odontologia. $\mathrm{Na}$ área tecnológica, foram 58 estudantes ( 11 mulheres e 47 homens), com média deidade de 19,38 $\pm 1,68$ anos, cursando Engenharia Civil, Engenharia Elétrica, Engenharia Química, Arquitetura e Urbanismo, Engenharia M ecânica ou Engenharia da Computação. Todos os estudantes foram voluntários.

Ao separar a amostra por sexo, as mulheres da área humanística apresentaram média de idade de $20,6 \pm 3,32$ anos e os homens desta área apresentaram média de idade de $21,89 \pm 7,68$ anos. $\mathrm{Na}$ área biomédica, as mulheres apresentaram média de idade de 19,84 $\pm 1,82$ e os homens apresentaram média de idade de $19,59 \pm 65$. Por fim, as mulheres da área tecnológica apresentaram média de idade de 19,55 \pm 1 ,44 e os homens dessa área apresentaram média de idade de 19,34 $\pm 1,75$ anos.

\section{Protocolos}

Utilizou-se um termo deconsentimento livree esclarecido, conten do al gumas informações a respeito do estudo realizado, tais como seu objetivo, sua natureza, benefícios, riscos e desconforto desencadeados pela partici pação nas atividades propostas, o grau de envolvimento dos participantes na investigação e seu caráter de confidencialidade. Para conseguir dados sobre os estudantes, fez-se uso deuma ficha de identificação, a ser preenchida por eles, que continha questionamentos sobre dados pessoais, seus horários de aula e horários das atividades extracurriculares. 0 Inventário de Ansiedade Traço-Estado (IDATE) também foi aplicado e consiste em um questionário de auto-avaliação que objetiva medir o estado de ansiedade (níveis reais de intensidade de ansiedade) e traço de ansiedade (tendência a reagi i à pressão psicológica com diferentes graus de intensidade). Esse inventário possui quarenta afirmações, sendo vinte para a escala de estado de ansiedade e vinte para a de traço de ansiedade.

\section{Procedimentos}

\section{Coleta de dados}

Após aprovação da pesquisa pelo comitêde ética em pesquisas da U FRN, estabeleceu-se um contato prévio com a coordenação de cada curso, a fim de explicar sobrea natureza da pesquisa e solicitar a autorização para realizar a aplicação dos instrumentos nas salas de aula. Em cada um dos cursos, foi solicitada a participação voluntária de dez alunos. A estes alunos foram entregues os protocolos da investigação acima citados, havendo a leitura inicial do termo de consentimento livre esclarecido, seguida pelo preenchimento da ficha de identificação e, por último, foi respondido o IDATE.

Análise de dados

A pós o término da coleta de dados e da posterior avaliação da ficha deidentificação e do IDATE, deu-se início à organização dos dados obtidos, separando-os por área de conhecimento e por sexo.

$\mathrm{Na}$ avaliação do IDATE, são atribuídos pesos a cada um dos vinte itens de cada subescala do IDATE (a escala de ansiedade traço e a de ansiedadeestado), de maneira que os pesos são iguais ao valor da avaliação a ser atribuída (que pode ser 1 , 2, 3 ou 4) para os itens em que uma alta avaliação 
( 3 ou 4) indique uma alta ansiedade; e os pesos são invertidos $(4,3,2,1)$ nositens em que uma alta avaliação indiqueuma baixa ansi edade. Caso o indivíduo tivesse omitido um ou dois itens na escala de ansiedade-traço ou na de ansiedade-estado, sua avaliação proporcional era obtida com a determinação do valor médio dos itens respondidos, a multiplicação desse valor por vinte e, em seguida, seu arredondamento para o número mais alto seguinte. A amplitude de escores possíveis para cada uma das subescalas varia de um mínimo de vintea um máximo de oitenta.

Assim, o IDATE estabelece como média de ansiedade-traço para alunos do ciclo básico o valor de 40,16 $\pm 9,70$, ecomo média de ansiedade-estado o valor de 40,04 $\pm 10,13$. 0 IDATE também fornece o valor médio de ansiedade- traço e de ansiedadeestado separados por sexo. Para estudantes do ciclo básico do sexo feminino, a média de ansiedade-traço equivale a $41,67 \pm 10,14$ e para o sexo masculino essa média corresponde a 38,64 $\pm 9,25$. Quanto à ansiedade-estado, o valor médio estabelecido para estudantes do ciclo básico do sexo feminino éde $41,09 \pm 10,81$ e para o sexo masculino éde 38,99 $\pm 9,45$.

Em seguida, os escores de ansiedade de cada área foram submetidos a uma análise estatística através do teste $t$ de Student para amostras independentes, que permite verificar se existem diferenças estatisticamente significantes entre os escores médios gerais de traço e estado de ansiedade das três áreas de conhecimento da UFRN, e entre os escores médios separados por sexo de cada área. As correlações que foram consideradas estatisticamente significantes possuíam um $p<0,05$.

\section{Resultados}

Os resultados encontrados referem-se aos escores médios de ansiedade-traço eansiedade-estado dos estudantes do ciclo básico das áreas humanística, biomédica e tecnológica da U FRN .

Ao avaliar os resultados do IDATE dos 53 sujeitos da área humanística, observou-se que estes alunos obtiveram um escore médio de traço de ansiedade de $41,06 \pm 13,43$, quese encontra dentro da média de ansiedade traço referente a estudantes do ciclo básico, a qual é 40,16 $\pm 9,70$. Quanto aosescores médios de ansiedade estado, essa amostra obteve a média de 39,98 $\pm 13,94$, dado este que está dentro da média esperada para estudantes do ciclo básico, a qual é 40,04 $\pm 10,13$ (Figura 1).

Ao analisar por sexo os dados obtidos, depara-se com escores médios de traço e estado de an- siedade para as estudantes do sexo feminino de $43,77 \pm 12,90$ e $42,86 \pm 14,15$ respectivamente, e para os estudantes do sexo masculino a média de ansiedade-traço foi de 35,78 $\pm 13,20$ e de ansiedade estado foi 34,39 $\pm 12,01$ (Figura 2). Esses resultados demonstram que tanto as mulheres quanto os homens da área humanística possuem escores médios detraço eestado de ansiedade equivalentes aos escores esperados no ciclo básico universitário para as mulheres (ansiedade traço, 41,67 $\pm 10,14$ e ansiedade-estado, $41,09 \pm 10,81$ ) epara os homens (ansiedade- traço, 38,64 $\pm 9,25$ e ansiedade-estado, $38,99 \pm 9,45$ ).

$\mathrm{Na}$ área biomédica, observou-se que os 47 alunos obtiveram um escore médio de traço de ansiedade de $42,49 \pm 14,95$, que se enquadra na média de traço de ansiedade referente a estudantes do ciclo básico $(40,16 \pm 9,70)$. Q uanto aos escores de ansiedade-estado, a amostra obteve a média de $43,32 \pm 12,52$, dado este que também se encontra dentro do esperado para os estudantes do ciclo básico $(40,04 \pm 10,13)$ (Figura 1 ).

Separados por sexo, tem-se escores médios de $42,08 \pm 15,82$ para traço de ansiedade e 42,48 \pm 11,70 para estado de ansiedade nas estudantes da área biomédica. Para os estudantes do sexo masculino dessa área, as médias de traço e estado de ansiedade foram $42,95 \pm 14,25$ e 44,27 $\pm 13,61$ respectivamente (Figura 2). Diante disso, percebe-se que os escores encontrados estão dentro da média esperada, tanto para mulheres quanto para homens do ciclo básico universitário.

Ao analisar os dados de ansiedade na área tecnológica, observou-se que os 58 alunos dessa área obtiveram um escore médio de traço de ansiedade de $36,50 \pm 11,47$, queestá dentro da média de traço de ansiedade referente a estudantes do ciclo básico universitário. Quanto aos escores de ansiedadeestado, a amostra obteve a média de $39,40 \pm 13,49$, dado este que também se encontra dentro da mé dia esperada para estudantes do ciclo básico (Figura 1).

Quando separados por sexo, tem-se escores médios de ansiedade-traço para as estudantes da área tecnológica de 39,91 $\pm 10,67$, o que significa que estas mulheres obtiveram um escore médio equivalente ao esperado para as mulheres do ciclo básico. Quanto ao seu escoremédio de ansiedadeestado, estefoi 39,91 $\pm 14,19$, quetambém equivale à média esperada para as mulheres do ciclo básico. Os estudantes do sexo masculino da área tecnológica obtiveram o escore médio de ansiedade traço de $35,7 \pm 11,61$, e de ansiedade estado de 39,28 \pm 13,48 , os quais se encontram dentro das médias para os homens do ciclo básico (Figura 2). 


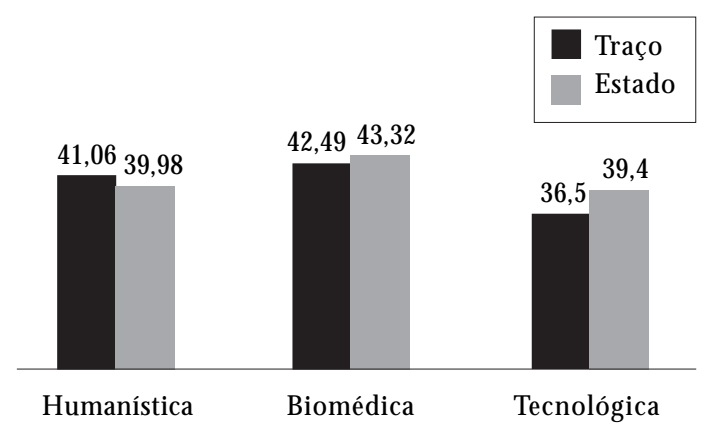

Figura 1. Escores médios de traço e estado de ansiedade em estudantes do ciclo básico da UFRN.

Fonte: Dados de pesquisa realizada em outubro de 2004, no campus central da UniversidadeFederal do Rio Grande do Norte, Natal, RN .

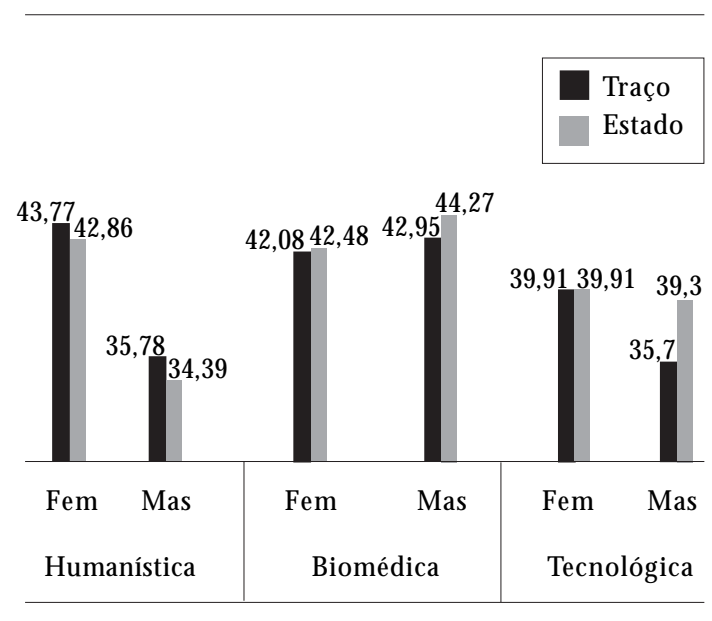

Figura 2. Escores médios de traço e estado de ansiedade separados por área de conhecimento e por sexo.

Fonte: Dados de pesquisa realizada em outubro de 2004, no campus central daU niversidadeFederal do Rio Grande do Norte, Natal, RN .

Ao analisar a ficha de identificação, observouse que as áreas humanística e tecnológica apresentam carga-horária reduzida, pois assistem aulas em apenas um turno durante 0 dia. Nessas áreas, também se encontrou uma significativa quantidade de alunos que trabalham ou desempenham al- gum outro tipo de atividade diária, como cursos de idiomas ou até mesmo aulas em uma outra faculdade.

Os estudantes da área biomédica apresentaram as demandas acadêmicas mais intensas, provenientes das aulas diárias que os alunos assistem em dois turnos, das monitorias e das aulas extras oferecidas nosfinais desemana. Também se encontrou na área biomédica o dado de que aproximadamente metade da amostra realiza algum tipo de atividade fora da universidade, como cursos de idiomas, que se somam às suas demandas acadêmicas.

Ao observar os altos desvios-padrão encontrados nos resultados, percebe-se que, apesar dos escores médios de ansi edade traço e ansiedade-estado dos estudantes das áreas biomédica, humanística e tecnológica terem se enquadrado na média de ansiedade esperada para o perfil da amostra (adultos jovens e estudantes do ciclo básico), há alguns estudantes, dentro desses grupos, que apresentaram escores tanto de traço quanto de estado de ansiedade acima da média (Tabela 1). Além de observar as médias individuais por área, percebeu-se que os escores médios de ansiedade separados por sexo também apresentaram altos desvios-padrão, levando à observação da quantidade de homens e mulheres de cada área que possuíam escores individuais abaixo, dentro e acima da média esperada para estudantes do sexo masculino e do sexo feminino do ciclo básico (Tabela 2).

Quando se objetivou avaliar se existiam diferenças estatisticamente significantes entre as áreas e entre os sexos para ansiedade-traço e ansiedadeestado, de todas as correlações possíveis que foram estabelecidas, apenas três foram consideradas estatisticamente significantes. A primeira diferença ocorreu entre as áreas biomédica e tecnológica $(t=2,322 ; p<0,05)$ para os escores médios de traço de ansiedade, sugerindo que os estudantes de biomédica são mais ansiosos. A segunda ocorreu entre os escores médios de ansiedade-estado dos estudantes do sexo masculino das áreas humanística e biomédica $(t=-2,407 ; p<0,05)$, sugerindo que os estudantes de biomédica estão numa situação mais ansiogênica de que os estudantes de humanística. E aúltima diferença estatisticamente significante foi observada entre as áreas de conhecimento tecnológica ebiomédica ( $t=-2,246 ; p<0,05$ ) e está associada aos escores médios de ansiedadetraço dos estudantes do sexo masculino, mostrando que esses estudantes de biomédica são mais ansiosos (Tabela 3). 
Tabela 1. Distribuição das médias individuais de traço e estado de ansiedade por área.

\begin{tabular}{|c|c|c|c|c|c|c|}
\hline & \multicolumn{2}{|c|}{ Humanística } & \multicolumn{2}{|c|}{ Biomédica } & \multicolumn{2}{|c|}{ Tecnológica } \\
\hline & Traço & Estado & Traço & Estado & Traço & Estado \\
\hline Acima da média & $34 \%$ & $36,2 \%$ & $30,2 \%$ & $30,2 \%$ & $15,5 \%$ & $29,3 \%$ \\
\hline Dentro da média & $31,3 \%$ & $19,1 \%$ & $28,3 \%$ & $30,2 \%$ & $39,7 \%$ & $29,3 \%$ \\
\hline Abaixo da média & $44,7 \%$ & $44,7 \%$ & $41,5 \%$ & $39,6 \%$ & $44,8 \%$ & $41,4 \%$ \\
\hline
\end{tabular}

Fonte: Dados de pesquisa realizada em outubro de 2004, no campus central da U niversidadeFederal do Rio Grandedo Norte, $N$ atal, RN.

Tabela 2. Distribuição das médias individuais de traço e estado de ansiedade por sexo em cada área.

\begin{tabular}{|c|c|c|c|c|c|c|c|c|c|}
\hline \multirow{3}{*}{$\begin{array}{c}\text { Distribuição } \\
\text { da média }\end{array}$} & \multicolumn{4}{|c|}{ Biomédica } & \multicolumn{3}{|c|}{ Humanística } & \multicolumn{2}{|c|}{ Tecnológica } \\
\hline & \multicolumn{2}{|c|}{ Feminino } & \multicolumn{2}{|c|}{ M asculino } & \multicolumn{2}{|c|}{ Feminino } & M asculino & Feminino & Masculino \\
\hline & Traço & Estado & Traço & Estado & Traço & Estado & Traço Estado & Traço Estado & Traço Estado \\
\hline Acima & $28 \%$ & $28 \%$ & $45,4 \%$ & $54,5 \%$ & $34,3 \%$ & $37,1 \%$ & $16,7 \% \quad 16,7 \%$ & $27,3 \% 45,4 \%$ & $21,3 \% \quad 31,9 \%$ \\
\hline Dentro & $48 \%$ & $52 \%$ & $36,4 \%$ & $27,3 \%$ & $40 \%$ & $37,1 \%$ & $33,3 \% \quad 44,4 \%$ & $54,5 \% 27,3 \%$ & $40,4 \% \quad 38,3 \%$ \\
\hline Abaixo & $24 \%$ & $20 \%$ & $18,2 \%$ & $18,2 \%$ & $25,7 \%$ & $25,8 \%$ & $50 \% \quad 38,9 \%$ & $18,2 \% 27,3 \%$ & $38,3 \% \quad 29,8 \%$ \\
\hline
\end{tabular}

Fonte: Dados de pesquisa realizada em outubro de 2004, no campus central da UniversidadeFederal do Rio Grandedo N orte, $N$ atal, RN.

Tabela 3. Comparação entre médias de traço e estado de ansiedade por área e sexo (Test t; $p<0,05$ ).

\begin{tabular}{|c|c|c|c|c|c|c|}
\hline & \multicolumn{2}{|c|}{ H umanística*Biomédica } & \multicolumn{2}{|c|}{ Humanística*Tecnológica } & \multicolumn{2}{|c|}{ Biomédica*Tecnológica } \\
\hline & $\mathrm{t}$ & $\mathrm{p}$ & $\mathrm{t}$ & $\mathrm{p}$ & $\mathrm{t}$ & $\mathrm{p}$ \\
\hline Ansiedade-estado/Geral & 0,623 & NS & 0,224 & NS & 0,465 & NS \\
\hline Ansiedade-traço/Geral & $-0,505$ & NS & 1,927 & NS & 2,322 & 0,05 \\
\hline Ansiedade-estado/Feminino & 0,109 & NS & 0,602 & NS & 0,569 & NS \\
\hline Ansiedade-estado/M asculino & $-2,407$ & 0,05 & $-1,346$ & NS & 1,430 & NS \\
\hline Ansiedade-traço/ Feminino & 0,455 & NS & 0,899 & NS & $-0,414$ & NS \\
\hline Ansiedade-traço/M asculino & $-1,637$ & NS & 0,023 & NS & $-2,246$ & 0,05 \\
\hline
\end{tabular}

Fonte: Dados de pesquisa realizada em outubro de 2004, no campus central da Universidade Federal do Rio Grande do Norte, Natal, RN .

\section{Discussão econclusão}

0 objetivo da presente pesquisa foi avaliar a ansiedade traço e ansiedade-estado dos estudantes do ciclo básico em vários cursos das áreas humanística, tecnológica e biomédica da Universidade Federal do Rio Grande do Norte (UFRN), avaliando se existiam diferenças entre os escores de ansiedade dessas áreas e entre os escores de ansiedade separados por sexo.
Os resultados mostraram que os estudantes universitários do ciclo básico das três áreas de conhecimento daUFRN apresentaram médias detraço de ansiedade (biomédica, $42,49 \pm 14,95$; humanística, 41,06 \$13,43; etecnológica, 36,50 $\pm 11,47$ ) dentro da média esperada para estudantes do ciclo básico. I sso sugere que esses estudantes parecem não interpretar a situação do ciclo básico como ansiogênica, não apresentando uma característica de personalidade ansiogênica. 
Quanto às médias de estado de ansiedade encontradas (biomédica, 43,32 $\pm 12,52$; humanística, $39,98 \pm 13,94$; etecnológica, $39,40 \pm 13,49$ ), observa-se que estas também estão dentro da média esperada para estudantes do ciclo básico. Isso indica que os estudantes do primeiro ano da UFRN parecem não estar encarando a situação de entrada na universidade como ansiogênica.

Apesar desses escores de ansiedade-estado eansiedade traço estarem dentro da média, observando os escores das três áreas, percebe-se que a área biomédica é a área que está e é ansiosa (ansiedadeestado e ansiedade traço). A fim de se verificar se existiam diferenças estatisticamente significantes entre as três áreas, utilizou-se o teste t de Student para amostras independentes. Os resultados mostraram que a área biomédica é mais ansiogênica (traço) quando comparada com a tecnológica.

A área biomédica, em comparação com as outras áreas, é a área em que os estudantes apresentaram demandas acadêmicas mais intensas, provenientes das aulas diárias que os alunos assistem em dois turnos, das monitorias e das aulas extras oferecidas nos finais de semana. Também se encontrou que aproximadamente metade da amostra realiza al gum tipo de atividade fora da universidade, como cursos de idiomas que se somam às suas demandas acadêmicas. Tal situação exaustiva, com demandas externas (atividades e horários) e internas (desempenho acadêmico) traz consigo um conflito e a exigência de um esforço de ajustamento e adaptação maior, surgindo, então, a necessidade de traçar estratégias que possibilitem a superação dessa situação ${ }^{9}$. N este esforço de adaptação, o indivíduo com personalidade ansiosa poderá interpretar essa situação como ameaçadora, aumentando seu nível de ansiedade, por exceder seus recursos de enfrentamento. É importante frisar que a ansiedade traço ou disposicional éinfluenciada pelas situações, mas os dados estatísticos não encontraram evidências para ansiedade estado.

Ao dividir a amostra de cada área de conhecimento por sexo, percebe-se que as médias de ansiedade-traço, tanto dos estudantes do sexo feminino (biomédica, 42,08 $\pm 15,82$; humanística, 43,77 \pm 12 ,90; e tecnológica, $39,91 \pm 10,67)$ quanto do masculino (biomédica, 42,95 $\pm 14,25$; humanística, 35,78 $\pm 13,20$; etecnológica, 35,70 $\pm 11,61$ ), continuam dentro da média esperada para mulherese homens estudantes do ciclo básico universitário.

0 mesmo ocorre entre os escores médios de ansiedade-estado entre as mulheres (biomédica, $42,48 \pm 11,70$; humanística, $42,86 \pm 14,15$; etecnológica, 39,91 $\pm 14,19$ ) eentreos homens ( biomédi-

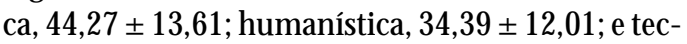

nológica, 39,28 $\pm 13,48$ ), que também se encontram dentro da média de estado de ansiedade esperada para mulheres e homens do ciclo básico.

Esses dados demonstram que tanto as estudantes do sexo feminino quanto os estudantes do sexo masculino parecem não apresentar a ansiedade disposicional, que transcende a barreiras de lugar e tempo, nem a ansiedade situacional no período estudado. Mas, observando os escores, os resultados sugerem que as estudantes de biomédica e humanística, e os estudantes de biomédica são e estão ansiosos. Esses dados, em conjunto com os dados encontrados estatisticamente de ansiedadetraço para a amostra geral (biomédica sendo mais ansiosa), corroborariam a idéia de que o aumento de ansiedade disposicional (traço) seria determinado em parte pela el evação da ansiedade situacional (estado), pois os escores de ansiedade-traço e ansiedade-estado dos estudantes de ambos os sexos de biomédica e das estudantes de humanística estão elevados. Essa idéia também é confirmada pelos dados de ansiedade traço dos estudantes do sexo masculino de humanística que não estavam elevados e nem os de ansiedade-estado.

Com o intuito de verificar estatisticamente os dados acima comentados, utilizou-se o teste $t$ de Student. Foram encontradas diferenças estatisticamente significantes entre os escores de ansiedade-estado para os estudantes do sexo masculino das áreas de biomédica e humanística ( $\mathrm{t}=-2,407$; $p<0,05)$, e entre os escores de traço de ansiedade para os homens de biomédica e tecnológica ( $\mathrm{t}=$ 2,$246 ; p<0,05$ ), sugerindo que os homens de biomédica estão e são mais ansiosos que as outras duas áreas.

Apesar de existirem vários estudos que demonstrem a prevalência desintomas e/ou detranstornos de ansiedade entre as mulheres por exercerem mais funções que os homens ${ }^{2,6,11}$, existem estudos que não observaram diferenças significativas entre a presença de distúrbios emocionais entre homens e mulheres ${ }^{15-17}$.

Na presente pesquisa, os dadosmostraram, pela primeira vez, que os homens estão e são mais ansiosos que as mulheres. Esses dados parecem sugerir que a situação vivenciada na área biomédica, pelo que já se comentou, é a mais ansiogênica; porém, como as mulheres enfrentam mais situações ameaçadoras (as mulheres brasileiras vivem numa estrutura social machista na qual têm de enfrentar maiores obstáculos para ingressar em uma carreira quelhepermiteuma maior independência social e econômica, além de ser designado o papel social de dona de casa), elas possuem um maior número demecanismos deenfrentamento, ao contrário dos 
homens que estão iniciando a faculdade (contexto novo) e estão lidando com situações que para eles são diferentes daquel as que el es estão acostumados a lidar, exigindo mecanismos diferentes.

Analisando os dados da amostra geral, percebe-se que os estudantes da área biomédica e humanística estão acima da média, e que, em especial, os estudantes de biomédica estão mais ansiosos (estado de ansiedade elevado). Esse dado corrobora mais uma vez a idéia de que a situação que os estudantes de biomédica estão vivenciando parece aumentar sua ansiedade disposicional.

Procedendo-se à análise por sexo destes grupos em relação aos desvios-padrão, a fim de compreender as diferenças estatísticas encontradas para os homens, observou-se que, na área humanística, $16,7 \%$ dos homens da amostra apresentaram escores de ansiedade-estado acima da média, e, na área tecnológica, 21,3\% dos estudantes do sexo masculino apresentaram escores de ansiedade traço acima da média. Voltando-se à área biomédica, percebe-se que $54,5 \%$ dos homens apresentaram escores de ansiedade-estado acima da média e que $45,4 \%$ desses homens apresentaram escores de ansiedade traço acima da média esperada para essa população. Esses percentuais parecem propiciar as diferenças estatisticamente significantes encontradas para os homens, além de que a quantidade de homens com traço de ansiedade acima da média pode explicar a elevação dos escores de ansiedadeestado dos mesmos.

Isso acaba por confirmar a idéia exposta em vários estudos ${ }^{6,11,18,19}$, na qual a elevação da ansiedade-estado pode ser ocasionada pelas estruturas depersonalidade, que influenciam a maneira como o indivíduo percebe uma situação ou evento estressor e como reagirá a ele. Dentre estas estruturas, estão os escores de ansiedade-traço que podem ser excelentes preditores dessa reação.

Assim, as análises realizadas mostraram que a situação de entrada na universidade, dependendo da formulação da grade curricular, dos horários disponibilizados para estes alunos e do número de demandas, pode se configurar como um período de transição considerado ameaçador, cuja estrutura do ciclo básico poderá aumentar a ansiedade nesse momento. Além disso, deve-se considerar as características individuais, a maneira como cada um percebe os eventos que vivencia, atribuindo a eles valores positivos ou negativos, uma vez que esta avaliação está baseada na sua personalidade e no seu histórico de vida. 


\section{Colaboradores}

CL Ferreira auxiliou na elaboração e na execução do estudo e contribuiu para a redação e revisão final do artigo; KM Almondes contribuiu com a elaboração do estudo, a análise e revisão crítica do artigo; ANS M ata, CA Lemos e LP Braga participaram igualmente da execução do estudo e concepção teórica do artigo; eEM C M aia auxiliou no delineamento do estudo e na redação final do artigo.

\section{Referências}

1. Almondes KM. Padrão do ciclo sono vigília e sua relação com a ansiedade em estudantes universitários [dissertação]. Natal (RN): Universidade Federal do Rio Grande do Norte; 2001.

2. Holmes DS. Psicologia dos transtornos mentais. Porto Alegre: Artes M édicas; 1997.

3. Twenge JM. The age of anxiety? Birth cohort change in anxiety and neurocitism, 1952-1993. Journal of Personality and Social Psychology 2000; 79:1007-1021.

4. Davidson J, Connor KM. Social anxiety disorder: a treatable condition. Drug Benefit Trends 1999; 11:1-4.

5. Robins LN, Helzer JE, Weissman M M, Orvaschel $H$, Gruenberg E, Burke JD, Reigier DA. Life-time prevalence of specific psychiatric disorders in three sites. Archives of General Psychiatric 1984; 41:949-958.

6. La Rosa J. Ansiedade, sexo, nível sócio-econômico e ordem de nascimento. Psicologia: Reflexão e Crítica 1998;11(1):59-70.

7. Oliveira KL, Santos AAA, Cruvinel M, Neri AL. Relação entre Ansiedade, Depressão e Desesperança entre Grupos de Idosos. Psicologia em Estudo 2006; 11(2):351-359.

8. Silver HK. Medical student and medical school. Jamaica 1982; 247:304-320.

9. Caplan G. Princípios de psiquiatria preventiva. Rio de Janeiro: Zahar Editores; 1980.

10. Papalia DE, Olds SW. Desenvolvimento humano. Porto Alegre: Artes M édicas Sul; 2000.

11. Spielberger CD, Gorsuch RL, Lushene RE. Inventário de Ansiedade Traço-Estado - IDATE. Rio de Janeiro: Cepa; 1979.

12. Almondes KM, Medeiros ALD, Lima PF, Rolim SAM, Dias Júnior SA, Araújo JF. Ansiedade em estudantes de medicina: uma realidade desnecessária. Revista Saúde 2002; 16(1):17-24.

13. Ferreira CL. A ansiedade em estudantes universitários: a realidade do curso de psicologia [relatório de pesquisa]. Natal (RN): Universidade Federal do Rio Grande do Norte; 2003.

14. M eleiro AMAS. 0 médico como paciente. São Paulo: Lemos Editorial; 2001.

15. Benítez CG, Quintero JB, Torres RB. Prevalence of risk for mental disorders among undergraduate medical students at the Medical School of the Catholic University of Chile. Rev Med Chil 2001 Fev; 129(2):173-178.

16. M iller PM CC, Surtees PG. Psychological symptoms and their course in first-year medical students as assessed by the Interval General Health Questionnaire (I-GHQ). Br J Psychiatry 1991; 159:199-207.

17. Souza FGM, M enezes MGC. Estresse nos Estudantes de Medicina da Universidade Federal do Ceará. Revista Brasileira de Educação M édica 2005; 29(2):91-96.

18. Hemenover SH, Dienstbier RA. Prediction of $\mathrm{H}$ ealth Patterns from General Appraisal, Attributions, Coping, and Trait Anxiety. Motivation and Emotion 1998; 22(3):231-253.

19. Lazarus RS, Folkman S. Stress, Coping and Adaptation. New York: Springer; 1984.

Artigo apresentado em 29/05/2006

Aprovado em 27/02/2007

Versão final apresentada em 30/04/2007 